\title{
REVisão deliteratura Comorbidade entre transtorno dismórfico corporal e transtornos alimentares: uma revisão sistemática
}

\author{
Comorbidity between body dysmorphic disorder and eating disorders: \\ a systematic review
}

Antonio Leandro Nascimento', Marcos Muniz Moreira', Juliano Victor Luna', Leonardo F. Fontenelle,'2

\section{RESUMO}

Introdução: A insatisfação com a imagem corporal é uma das características mais proeminentes do transtorno dismórfico corporal (TDC) e dos transtornos alimentares (TA). Esses dois grupos de transtornos apresentam semelhanças em suas características psicopatológicas e epidemiológicas e na resposta ao tratamento. Apesar de ambos serem considerados transtornos do espectro obsessivo-compulsivo, pouco se sabe em relação à comorbidade entre esses dois grupos de transtornos. Objetivo: Avaliar a produção bibliográfica atual sobre a comorbidade entre TDC e TA. Método: Foi realizada uma revisão sistemática nos bancos de dados PubMed e ISI Web of Science dos artigos referentes à comorbidade entre TDC e TA. Resultados: Oito artigos foram selecionados. As amostras de portadores de TA apresentavam

\section{Palavras-chave}

Transtorno dismórfico corporal, transtornos alimentares, transtornos do espectro obsessivocompulsivo, comorbidade. comorbidade com TDC em $6 \%$ a 39\% dos casos. Dos pacientes com TDC, 10\% a 32,5\%, por sua vez, podem apresentar comorbidade com TA. O TDC frequentemente precedia o TA, e os pacientes com comorbidade apresentavam quadro clínico mais grave que os com apenas um dos transtornos. Conclusão: Estudos recentes demonstram que as taxas de comorbidade entre esses dois transtornos são elevadas. Apesar disso, o TDC raramente é diagnosticado em portadores de TA, mesmo quando é o transtorno que causa mais incômodo.

\begin{abstract}
Background: Body image dissatisfaction is one of the most prominent psychopathological features of body dysmorphic disorder (BDD) and eating disorders (ED). These groups of disorders present similarities in psychopathological and epidemiological features and also in response to treatment. Although these disorders are included in the obsessive-compulsive spectrum, little is known about the comorbidity between the two groups of disorders. Objective: To evaluate the current bibliographic production on the comorbidity of BDD and ED. Method: A systematic review has been conducted on the PubMed and ISI Web of Science databases. Results: Eight articles have been selected. These articles report that patients with ED may present comorbidity with BDD in $6 \%$ to $39 \%$ of the cases. $10 \%$ to $32.5 \%$ of the patients with BDD might present comorbidity with a TA. BDD preceded ED in the majority of the patients with comorbidity and patients with both disorders had more severe presentations than patients with either disorder alone. Conclusion: Recent studies have shown that comorbidity ratios between these disorders are high. However, BDD is seldom diagnosed in patients with $E D$, even when it is the most severe disorder.
\end{abstract}

\section{Keywords}

Body dysmorphic disorder, eating disorders, obsessivecompulsive spectrum disorders, comorbidity.

Recebido em
$19 / 2 / 2010$
Aprovado em
$5 / 3 / 2010$

1 Universidade Federal do Rio de Janeiro (UFRJ), Instituto de Psiquiatria (IPUB), Programa de Pós-graduação em Psiquiatria e Saúde Mental (PROPSAM).

2 Universidade Federal do Rio de Janeiro (UFRJ), Faculdade de Medicina, Departamento de Psiquiatria e Medicina Legal.

Endereço para correspondência: Antonio Leandro Nascimento

Av. Nossa Senhora de Copacabana, 1066, sala 706, Copacabana - 22060-030 - Rio de Janeiro, RJ

E-mail: antonio.leandro.nascimento@gmail.com 


\section{INTRODUÇÃO}

Diversos transtornos mentais podem causar, ao longo de sua evolução, incômodo com a forma corporal. A insatisfação com a forma corporal é das características psicopatológicas mais proeminentes do transtorno dismórfico corporal (TDC) e dos transtornos alimentares (TA).

Apesar de os primeiros relatos de casos de pacientes com anorexia nervosa (AN) 1,2 não mencionarem incômodo com a imagem corporal, detendo-se apenas nas alterações dos hábitos alimentares e do peso das pacientes, ainda no século XIX e no início do século XX, Jean Martin Charcot descreveu a ideia fixa de obesidade (idée fixe d'obésité) como um aspecto psicopatológico central da $\mathrm{AN}^{3}$. No artigo em que descreveu a bulimia nervosa (BN) como uma variante da AN, Russel ${ }^{4}$ relata que as pacientes apresentavam "medo mórbido de engordar" como um dos principais sintomas.

$\mathrm{Na}$ descrição original do TDC ${ }^{5}$, a dismorfofobia (o termo originalmente utilizado para denominar o TDC) foi descrita como "a consciência da própria deformidade, o indivíduo teme ser ou tornar-se deformado".

Os sistemas classificatórios atuais descrevem a AN como um transtorno caracterizado por perda de peso intencional associada a uma psicopatologia específica, que envolve medo de engordar e de ter uma silhueta arredondada, manutenção de baixo peso e comumente desnutrição de grau variável acompanhada de modificações endócrinas e metabólicas secundárias. Os sintomas compreendem uma restrição das escolhas alimentares, a prática excessiva de exercícios físicos, vômitos provocados e a utilização de laxantes, anorexígenos e de diuréticos ${ }^{6}$.

Já a BN é caracterizada por "episódios de hiperfagia" e "pavor mórbido de engordar"6 ou como "episódios regulares de compulsão alimentar" e "autoavaliação influenciada pelo peso corporal"7, salientando que o incômodo corporal nos TA está fundamentalmente ligado ao peso e às características por ele influenciadas.

O TDC é descrito atualmente como "uma preocupação persistente com uma suposta deformidade ou desfiguramento"6 ou como "uma preocupação com um defeito imaginário na aparência ou uma preocupação excessiva com um defeito físico discreto"7 quando não melhor explicados por outro transtorno.

A presença de pensamentos recorrentes sobre a imagem corporal nos dois grupos de doenças e de comportamentos repetitivos (contagem de calorias e comportamentos compensatórios nos TA e verificação, busca por reasseguramento e estratégias de camuflagem no TDC) levou esses dois transtornos a serem incluídos no grupo dos transtornos do espectro obsessivo-compulsivo (TEOC) ${ }^{8}$. As semelhanças na fenomenologia, no padrão de comorbidades, nas características genéticas e familiares, no envolvimento de circuitos cerebrais e na resposta ao tratamento aproximam ainda mais esses transtornos.

A prevalência de AN ao longo da vida em mulheres é estimada entre $0,3 \%$ e $1 \%{ }^{9}$, e os estudos indicam que a prevalência em homens chega, se muito, a 0,3\%. De acordo com os critérios atuais para o diagnóstico de bulimia nervosa, a prevalência desse transtorno em mulheres em idade reprodutiva é de $1 \%$ a $1,5 \%^{10,11}$. A maior parte dos pacientes com TA não preenche todos os critérios diagnósticos, sendo classificados como portadores de transtornos alimentares sem outra especificação (TA-SOE), cuja prevalência atinge 3\% a 5\% das mulheres de 15 a 30 anos em países ocidentais.

Estudos populacionais mostraram prevalência de 0,7\% a 1,7\% de TDC na população em geral. Estudos em populações que demonstram maior preocupação com a aparência física, como pessoas procurando tratamento dentário estético ou cirurgia plástica, encontraram prevalência de 3\% a 15\%. A razão entre pacientes do sexo feminino e masculino varia de 1:1 a 3:2, de acordo com o estudo ${ }^{12}$.

O padrão de comorbidades em pacientes com TA e TDC é bastante semelhante. A prevalência de transtorno obsessivocompulsivo (TOC) em pacientes com AN varia de $25 \%$ a $37 \%$, enquanto a prevalência de AN em pacientes com TOC varia de $9 \%$ a 26\% ${ }^{13}$. Em pacientes com TDC, a prevalência de TOC varia de $8 \%$ a 37,5\%, enquanto a prevalência de TDC em pacientes com TOC é estimada em 7,7\% ${ }^{13}$. Pacientes portadores de TDC e TA apresentam incômodo com a imagem corporal em graus semelhantes e também baixa autoestima ${ }^{14}$.

A expressão "quando não melhor explicada por outro transtorno", nos critérios para o diagnóstico do TDC, permite diagnosticar os dois transtornos quando um paciente portador de um TA apresenta incômodos com aspectos de sua imagem corporal. Os estudos atuais mostram que portadores de TA podem apresentar incômodos com seu corpo que não estão relacionados às variações no peso ${ }^{15-18} \mathrm{com}$ uma taxa de comorbidade que pode atingir os 39\%. Até 9\% dos pacientes com transtorno da compulsão alimentar periódica (TCAP), cujos critérios diagnósticos não incluem autoavaliação influenciada pela imagem corporal, apresentam comorbidade com TDC ${ }^{19}$. Assim, podem ficar incomodados com a cor dos olhos ou os cabelos, ou outro aspecto de sua aparência. Dessa forma, esses autores consideram que a comorbidade entre TA e TDC pode ser amplamente subestimada e que alguns portadores de TA podem apresentar períodos com sintomas de TDC antes do surgimento das alterações no comportamento alimentar.

\section{OBJETIVO}

Descrever a comorbidade entre TDC e TA baseado nos estudos já realizados sobre o tema. 


\section{MÉTODO}

Foi realizada uma revisão sistemática nos bancos de dados PubMed e ISI Web of Science. Os termos utilizados nas pesquisas foram "eating disorders" ou "anorexia nervosa" ou "bulimia nervosa" ou "binge eating disorder", combinados com "body dysmorphic disorder" ou "dysmorphophobia". Foram selecionados artigos que descrevessem estudos sobre a comorbidade entre esses dois transtornos, enfocando tanto aspectos como incidência e características clínicas quanto manifestações específicas, como a gravidade das manifestações de incômodo corporal.

\section{RESULTADOS}

Foram encontrados seis relatos de casos (um deles, comentado) e uma série de casos sobre o tema. Além das séries de casos, foram identificados cinco estudos sobre incômodo com a imagem corporal não relacionados ao peso em portadores de TA e três estudos sobre incidência de transtornos alimentares em pacientes com TDC.

As primeiras publicações sobre comorbidade entre TDC e TA são relatos de caso publicados em meados da década de 198020-23. Os três destacam a presença de incômodo com a imagem corporal não relacionado à forma e ao peso em períodos em que as pacientes já não apresentavam alterações do comportamento alimentar. Outro relato de dois $\operatorname{casos}^{24}$ também destaca a presença de incômodo com aspectos da imagem corporal não relacionados ao peso e menciona a possibilidade de dois diagnósticos. No ano seguinte, seria publicada uma série de $\operatorname{casos}^{25}$ de pacientes com AN que apresentaram, no início de seu quadro clínico, sintomas exclusivos de TDC. O primeiro estudo a avaliar sistematicamente o incômodo com a imagem corporal não relacionado ao peso em portadoras de transtornos alimentares ${ }^{26}$ comparou pacientes do sexo feminino com TA ( $n=25)$, pacientes com queixas relacionadas à imagem corporal que não preenchiam os critérios diagnósticos do DSM-III-R para TDC ou para qualquer TA ( $n=25)$ e funcionárias da universidade onde o estudo foi realizado $(n=25)$. As participantes foram avaliadas por meio de diversas escalas psicométricas para TA e TDC: Body Dysmorphic Disorder Examination (BDDE), Body Shape Questionnaire (BSQ), Multidimensional Body Relations Questionnaire (MBRQ) e Eating Disorder Examination (EDE). Os autores dividiram as queixas relacionadas à imagem corporal em relacionadas ao peso (ser muito gordo, muito grande, flácido, muito largo em geral ou em regiões específicas como barriga, coxas, nádegas ou flancos) e não relacionadas ao peso. A maior parte das pacientes com TA apresentou queixas secundárias sobre a imagem corporal e, para cerca de 30\% delas, essas queixas não estavam relacionadas ao peso (seios pequenos ou caídos, cicatrizes, manchas na pele, incômodo relacionado à forma do rosto e cabelo pouco cheio). Dentre as pacientes com TA, 88\% atingiram os escores necessários para o diagnóstico de TDC de acordo com o BDDE. Esse estudo mostrou que um maior grau de insatisfação corporal estava relacionado a um desejo maior de perder peso e que as pacientes com TA apresentavam incômodo com partes de seu corpo não relacionadas ao peso.

O segundo estudo que avaliou o incômodo com a imagem corporal não relacionado ao peso em portadores de transtornos alimentares comparou 53 portadoras de AN ou BN de acordo com os critérios da DSM-III-R a 73 mulheres selecionadas aleatoriamente da comunidade ${ }^{15}$. As pacientes foram avaliadas por meio de duas subescalas (Drive for thinness e Body Dissatisfaction) do Eating Disorders Inventory (EDI) e de uma lista em que poderiam marcar se apresentavam ou não incômodo com diversas partes de seu corpo. $\mathrm{O}$ estudo mostrou que as portadoras de TA apresentavam incômodos com diversas partes de seu corpo não relacionadas ao peso em frequência maior que os controles: pele (79,2\% vs. 52,1\%, $\mathrm{p}=, 002)$, dentes $(62,3 \%$ vs. 39,7\%, $\mathrm{p}=, 012)$, mandíbula (24,5\% vs. 9,7\%, $p=, 026)$, nariz (45,3\% vs. 24,7\%, $p=, 015)$, olhos $(22,6 \%$ vs. $12,3 \%, p=, 12)$, orelhas $(20,8 \%$ vs. $2,7 \%$, $\mathrm{p}=.001)$, cabelo $(52,8 \%$ vs. $39,7 \%, p=, 14)$ e altura $(28,3 \%$ vs. $13,7 \%, p=, 04)$. Assim como no primeiro estudo, as pacientes com maiores índices de incômodo corporal apresentavam maior desejo de emagrecer. Apesar desses achados, os autores não defendem a categorização desses pacientes como portadores de dois transtornos mentais, TA e TDC, preferindo atribuir o incômodo corporal não relacionado ao peso a deficiências egoicas, que seriam características dos TA.

O terceiro estudo com esse escopo ${ }^{16}$ foi realizado com o objetivo de pesquisar períodos de incômodo com a imagem corporal sem alterações no comportamento alimentar em pacientes portadoras de AN ( $n=36), B N(n=17)$ e controles $(n=40)$. Para isso, foram utilizados a Structured Clinical Interview for DSM-IV (SCID) e um questionário desenvolvido pelos autores para avaliar eventos de vida (rejeição por meninas da mesma idade, perda do interesse por meninos, perda de um parceiro, comentários negativos sobre a aparência da paciente ou comentários dos pais sobre a aparência da paciente). Esse estudo mostrou que $25 \%$ das portadoras de AN apresentaram, em um período que variou de três anos a seis meses antes do início das alterações do comportamento alimentar, sintomas de incômodo com a imagem corporal que poderiam ser diagnosticados como TDC. Nenhuma das pacientes com BN apresentou sintomas suficientes para o diagnóstico de TDC, porém 7,5\% apresentaram sintomas subclínicos. Nesse estudo, os autores defendem o diagnóstico dos dois transtornos (TA e TDC) nessas pacientes.

No quarto estudo de comorbidade entre TDC e TA ${ }^{17}$, foram investigados sintomas de incômodo corporal em 41 pacien- 
tes portadoras de AN, de acordo com os critérios diagnósticos da DSM-IV confirmados em uma entrevista pelo primeiro autor, usando o SCID. Foram utilizados o Body Dysmorphic Disorder Questionnaire, um questionário estruturado de preenchimento independente para avaliação de TDC, e o Brown Assessment of Beliefs Scale (BABS) para avaliação da intensidade das crenças referentes à imagem corporal. Das pacientes entrevistadas, 39\% apresentavam comorbidade com TDC. Essa comorbidade era mais frequente nas pacientes com AN do subtipo restritivo $(43,8 \%)$ que nas pacientes do subtipo purgativo (22,2\%). Nenhuma das pacientes portadoras de TDC foi diagnosticada por seu médico-assistente durante o tratamento da AN, mesmo aquelas que consideravam o TDC seu problema mais grave. Todas afirmaram que não mencionaram esse problema a seus médicos-assistentes por vergonha. Assim como no estudo polonês, o início dos sintomas de TDC precedeu o início da AN na maior parte das pacientes $(93,8 \%)$. Mais uma vez, as áreas que geravam maior incômodo estavam no rosto (nariz, pele, cabelo, queixo, lábios e olhos). Os pacientes com AN e TDC também apresentavam níveis mais baixos de funcionamento social e ocupacional, consumiam mais tempo de cada dia (e mais dias por ano) preocupados com sua aparência, foram hospitalizados maior número de vezes e havia um número maior de tentativas de suicídio nesse grupo. Além disso, mais de metade dos pacientes com AN e TDC buscara tratamentos cosméticos (cirurgias plásticas, tratamentos dermatológicos ou tratamentos dentários estéticos) a fim de tentar aliviar seu incômodo com a imagem corporal.

Em um estudo não controlado de pacientes com TDC ${ }^{27}$, os autores encontraram 10\% de comorbidade com transtornos alimentares. As regiões de maior incômodo corporal foram face (tamanho ou formato), pele, cabelos, nariz e constituição corporal e peso.

Em 2006, foi observada uma nova tendência nas publicações sobre TA e TDC: a pesquisa de preocupações com o peso e comorbidade com TA em portadores de TDC. No primeiro estudo realizado sobre 0 tema ${ }^{28}, 200$ pacientes portadores de TDC foram avaliados com o BDD Form, uma entrevista estruturada com 43 itens que auxilia na avaliação das áreas do corpo que causam incômodo, dos comportamentos relacionados à doença (como camuflagem e reasseguramento) e da presença de ideias de suicídio. Além do BDD Form, os participantes foram avaliados com as escalas BDDE, Yale-Brown Obsessive-Compulsive Scale modificada para transtorno dismórfico corporal (Y-BOCS-BDD), com a Brief Social Phobia Scale (BSPS), Quality of Life Enjoyment and Satisfaction Questionnaire (Q-LES-Q) e Social Adjustment Scale Self Report (SASSR). Dentre os 200 participantes, 29\% apresentavam preocupações relacionadas ao peso. O peso era a preocupação principal para apenas 3,5\% dos participantes.

Os pacientes foram divididos em grupos de TDC com preocupações relacionadas ao peso e sem preocupações re- lacionadas ao peso. Os pacientes com TDC e preocupações relacionados ao peso eram mais frequentemente mulheres e mais jovens. Elas apresentavam sintomas mais graves de acordo com o BDDE. Os pacientes com TDC e preocupações relacionadas ao peso apresentavam incômodo com áreas corporais não relacionadas ao peso em frequência maior que os sem preocupações relacionadas ao peso em quatro das cinco áreas de maior preocupação: pele (77.5 vs. 81.0\%, $p=0.169$ ), cabelo (62.1 vs. $55.6 \%, p=0.231$ ), nariz (48.3 vs. $35.2 \%, p=0.076)$, abdômen (55.2 vs. $32 \%, p=0.001$ ) e dentes ( 37.9 vs. $26.1 \%, p=0.141$ ). Portadores de TDC com incômodos relacionados ao peso também apresentavam maior incidência de sintomas de depressão, comorbidades psiquiátricas, tentativas de suicídio e pior funcionamento social.

No maior estudo sobre a comorbidade entre TDC e TA realizado até o momento (18), foram examinados 200 pacientes com TDC incluídos em um estudo longitudinal sobre TDC. Esse estudo empregou a maior bateria de avaliações padronizadas já utilizada em estudos desse tipo: Structured Clinical Interview for DSM-IV Non-patient Version (SCIDI/NP), BDDE, BDD Form, Y-BOCS-BDD, BABS, SAS-SR, Q-LES-Q, Longitudinal Internal Follow Up Evaluation Range of Impaired Funcioning Tool (Life-Rift), Global Assessment of Functioning Scale (GAF), Social and Ocupational Functioning Assessment Scale (SOFAS). Dos pacientes avaliados, 32,5\% apresentaram algum transtorno alimentar ao longo da vida (incluindo transtorno da compulsão alimentar periódica e outras variantes de transtorno alimentar sem outra especificação) e 9,5\% apresentaram um transtorno alimentar simultaneamente ao TDC. A maior parte dos pacientes com os dois transtornos $(63,1 \%)$ desenvolveu TDC antes da apresentação das alterações no comportamento alimentar. Os pacientes que apresentaram os dois transtornos ao longo da vida classificavam o TDC como o mais grave e apresentavam mais insatisfação com sua imagem corporal que os pacientes diagnosticados exclusivamente com AN.

O estudo mais recente a respeito da comorbidade entre TA e TDC investigou por meio do SCID a comorbidade psiquiátrica em uma amostra de 150 pacientes portadores de TCAP recrutados para um estudo caso-controle. Dentre os portadores de TCAP, o TA cujos critérios diagnósticos não incluem autoavaliação influenciada pela imagem corporal, $6 \%$ apresentavam comorbidade com TDC.

\section{DISCUSSÃO}

Os estudos revisados mostram que a comorbidade entre TA e TDC é frequente (6\% a 88\%) e que está relacionada a maior gravidade, maior índice de tentativas de suicídio e maior número de tratamentos frustrados. Os pacientes com AN e TDC também apresentam níveis mais baixos de funcionamento social e ocupacional, consomem mais tempo de cada dia 
(e mais dias por ano) preocupados com sua aparência e são hospitalizados mais frequentemente. Além disso, muitos desses pacientes buscam tratamentos cosméticos (cirurgias plásticas, tratamentos dermatológicos ou tratamentos dentários estéticos) a fim de tentar aliviar seu incômodo com a imagem corporal, apesar de já ter sido demonstrado que nesses pacientes a realização de cirurgias plásticas não traz alívio aos sintomas de incômodo com a imagem corporal. Apesar disso, na presença dos dois transtornos, o TDC frequentemente não é diagnosticado, mesmo quando considerado pelas pacientes a sua manifestação mais grave. Um dos motivos possíveis para essa ocorrência é a falta de investigação sistemática por parte dos clínicos de incômodos com a imagem corporal não relacionados à forma e ao peso em pacientes com TA. Mais estudos são necessários para determinar a eficácia de tratamentos combinados para esses dois transtornos e sua tolerabilidade por essa população.

\section{REFERÊNCIAS}

1. Lasègue C. On hysterical anorexia. Med Times Gazette. 1873;2:265-6.

2. Gull WW. Anorexia nervosa (Apepsia Hysterica, Anorexia Hysterica). Clinical Society's Transactions. 1874;7:22.

3. Habermas T. The psychiatric history of anorexia nervosa and bulimia nervosa: weight concerns and bulimic symptoms in early case reports. Int J Eat Disord. 1989:8(3):259-73.

4. Russel GFM. Bulimia nervosa: an ominous variant of anorexia nervosa. Psychol Med. 1979;9:429-48.

5. Morselli E. Sulla dismorfofobia e sulla tafefobia due forme non per anco descritte di pazzia com idee fisse. Bolletino della Accademia delle Scienze Mediche di Genova. 1891;6:110-9.

6. Organização Mundial da Saúde (OMS). Classificação Estatística Internacional de Doenças e Problemas Relacionados à Saúde - Décima revisão. São Paulo: Edusp; 1993. p. 1200.

7. American Psychiatric Association (APA). Diagnostic and Statistical Manual of Mental Disorders DSM-IV-TR Fourth Edition (Text Revision). Washington: American Psychiatric Publishing; 2000. p. 943.

8. McElroy SL, Phillips KA, Keck PE Jr. Obsessive compulsive spectrum disorder. J Clin Psychiatry. 1994;55:33-51.

9. Hoek HW, Van Hoeken D. Review of the prevalence and incidence of eating disorders. Int J Eat Disord. 2003;34(4):383-96.
10. Fairburn (G, Beglin SJ. Studies of the epidemiology of bulimia nervosa. Am J Psychiatry. 1990;147(4):401-8.

11. Keski-Rahkonen A, Hoek HW, Susser ES, Linna MS, Sihvola E, Raevuori A, et al. Epidemiology and course of anorexia nervosa in the community. Am J Psychiatry. 2007;164(8):1259-65.

12. Phillips K. The presentation of body dysmorphic disorder in medical settings. Prim Psychiatry. 2006;13(7):51-9.

13. Du Toit PL, Van Kradenburg J, Niehaus D, Stein DJ. Comparison of obsessive-compulsive disorder patients with and without comorbid putative obsessive-compulsive spectrum disorders using a structured clinical interview. Compr Psychiatry. 2001;42(4):291-300.

14. Rosen JC, Ramirez E. A comparison of eating disorders and body dysmorphic disorder on body image and psychological adjustment. J Psychosom Res. 1998;44(3-4):441-9.

15. Gupta MA, Johnson AM. Nonweight-related body image concerns among female eatingdisordered patients and nonclinical controls: some preliminary observations. Int J Eat Disord. 2000;27(3):304-9.

16. Jolanta JR, Tomasz MS. The links between body dysmorphic disorder and eating disorders. Eur Psychiatry. 2000;15:302-5.

17. Grant JE, Sim SW, Eckert ED. Body dysmorphic disorder in patients with anorexia nervosa: prevalence, clinical features and delusionality of body image. Int J Eat Disord. 2002;32:291.

18. Ruffolo JS, Phillips KA, Menard W, Fay C, Weisberg RB. Comorbidity of body dysmorphic disorder and eating disorders: severity of psychopathology and body image disturbance. Inst J Eat Disord. 2006;39(1):11-9.

19. Javaras KN, Pope HG, Lalonde JK, Roberts JL, Nillni YI, Laird NM, et al. Co-occurrence of binge eating disorder with psychiatric and medical disorders. J Clin Psychiatry. 2008:69(2):266-73

20. Cohen G. Anorexia nervosa and dysmorphophobia. Br J Psychiatry. 1987 Mar;150:406-7.

21. Jerome L. Anorexia nervosa or dysmorphophobia? Br J Psychiatry. 1987;150:560-1.

22. Sturmey P, Slade PD. Anorexia nervosa and dysmorphobia. A case study. Br J Psychiatry. 1986;149:780-2.

23. Pantano $M$, Santonastaso $P$. A case of dysmorphophobia following recovery from anorexianervosa. Int Jou Eat Disord. 1989;8(6):701-4.

24. Bulik CM, Sullivan PF. Two cases of body dysmorphic disorder in women with eating disorders. Eur Eat Dis Rev. 1997:5(2):96-101.

25. Rabe-Jabłońska J. [Liaison between body dysmorphic disorder and eating disorders]. Psychiatr Pol. 1998;32(2):155-63.

26. Rosen JC, Reiter J, Orosan P. Assessment of body image in eating disorders with the body dysmorphic disorder examination. Behav Res Ther. 1995;33(1):77-84.

27. Fontenelle LF, Telles LL, Nazar BP, De Menezes GB, Nascimento AL, Mendlowicz MV, et al. A sociodemographic, phenomenological and long-term follow up study of patients with body dysmorphic disorder in Brazil. Int J Psychiatry Med. 2006;36(2):243-59.

28. Kittler JE, Menard W, Phillips KA. Weight concerns in individuals with body dysmorphic disorder. Eat Behav. 2007;8(1):115-20. 